\title{
RESONANT TUNNELLING IN DOUBLE-BARRIER HETEROSTRUCTURES WITH AN ACCUMULATION LAYER
}

\author{
T. Wosiński, T. Figielski, A. MąKosa, S. Wrotek and W. Dobrowolski \\ Institute of Physics, Polish Academy of Sciences and College of Science \\ Al. Lotników 32/46, 02-668 Warszawa, Poland
}

\begin{abstract}
Two modes of electron gas injection in resonant tunnelling through $\mathrm{GaAs} / \mathrm{AlGaAs}$ double-barrier heterostructures were revealed while studying their current-voltage characteristics. Examining peculiarities of the characteristics within the temperature range $4-350 \mathrm{~K}$ and under a high magnetic field, we were able to distinguish the contribution to resonant tunnelling of ballistic-electrons injected from a three-dimensional electron gas in the emitter contact and that of electrons injected from a two-dimensional electron gas in the accumulation layer formed near the emitter barrier.
\end{abstract}

PACS numbers: $73.40 . \mathrm{Gk}, 73.50 . \mathrm{Jt}, 85.30 . \mathrm{Mn}$

The phenomenon of resonant tunnelling in a double-barrier heterostructure (DBH) has been expected to be very promising for the design of high speed electronic devices. In order to improve the performance of such devices, undoped spacer layers are often grown outside the active part of device to separate them from heavily-doped electrode layers thus preventing the incorporation of segregated dopant into this part of the structure during epitaxial growth. In such devices a weak shoulder appearing on the rising side of the resonant peak in the current-voltage characteristic has been observed in several cases [1-4]. It was attributed to resonant tunnelling from a three-dimensional electron gas (3DEG) of the emitter whereas the main resonance was due to $2 \mathrm{D}$ electrons tunnelling from quasi-bound states in the accumulation layer adjacent to the emitter barrier. In the present paper we report new results on temperature and magnetic field dependence of this feature, which give further evidence for the occurrence of the two modes of electron gas injection in resonant tunnelling.

We studied DBHs grown by molecular beam epitaxy on (100)-oriented $n^{+}$-GaAs substrates. The structures consisted basically of a $5.6 \mathrm{~nm}$ GaAs quantum well sandwiched between a $3.7 \mathrm{~nm}$ AlAs barrier and a $5.6 \mathrm{~nm} \mathrm{Al} \mathrm{Al}_{0.4} \mathrm{Ga}_{0.6} \mathrm{As}$ one. Heavily $n$-doped $\left(2 \times 10^{18} \mathrm{~cm}^{-3}\right)$ electrodes were separated from the barriers by spacer layers, with the donor concentration gradually decreased towards the barriers. They consisted of $50 \mathrm{~nm}$ of GaAs doped to $2 \times 10^{17} \mathrm{~cm}^{-3}, 50 \mathrm{~nm}$ of GaAs doped 
to $2 \times 10^{16} \mathrm{~cm}^{-3}$ and $5 \mathrm{~nm}$ of undoped GaAs. In our previous paper [3] we investigated DBHs composed of similar spacer layers but rather different double-barrier structure. The results we report here were obtained on devices of $25 \mu \mathrm{m}$ diameter defined by standard photolithography and mesa etching techniques.

We measured the current-voltage characteristics, $I(V)$, of the devices, using pseudo-four-probe technique and a variable dc voltage source. Simultaneously, we measured the first and the second derivatives, $\mathrm{d} I / \mathrm{d} V$ and $\mathrm{d}^{2} I / \mathrm{d}^{2}$, of the $I(V)$ characteristics using a probe signal at a frequency $\nu$ of about $1 \mathrm{kHz}$ and the peak-to-peak amplitude of $1 \mathrm{mV}$ and a lock-in detection at the frequency $\nu$ and $2 \nu$, respectively. The measurements were performed within the temperature range 4-350 $\mathrm{K}$ and under a magnetic field up to $13 \mathrm{~T}$.

The $I(V)$ characteristics display a resonant maximum in the tunnel current against bias voltage which end in a double-step feature (Fig. 1) similar to that frequently reported in the literature; cf. [5] and references therein. At a bias voltage of about $0.4 \mathrm{~V}$, just below that of the onset of the main resonance peak, an additional weak maximum appears at the characteristics (Fig. 1) which we called "precursor" of resonance [3]. It is more pronounced while measuring $\mathrm{d} I / \mathrm{d} V$ and $\mathrm{d}^{2} I / \mathrm{d} V^{2}$ against bias voltage, as shown in the insets in Fig. 1.

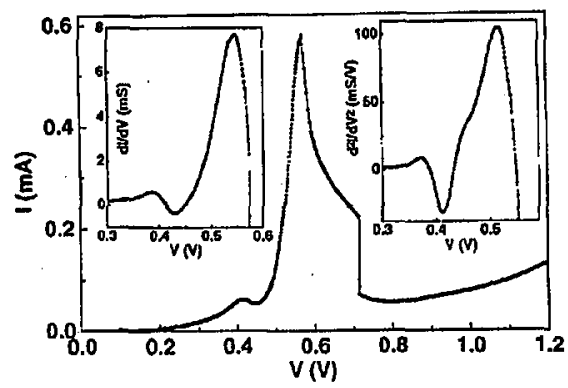

Fig. 1. Current-voltage characteristic of $25 \mu \mathrm{m}$ diameter device obtained from the investigated DBH measured at $150 \mathrm{~K}$. The insets show the first (left) and the second (right) derivatives of the $I(V)$ characteristic of the device measured in the range of increasing resonant-tunnelling current.

The precursor amplitude, defined as the difference between the relevant local maximum and minimum in the $\mathrm{d}^{2} \mathrm{I} / \mathrm{d} V^{2}$ curve multiplied by the difference of the voltages corresponding to the above extrema (see right inset in Fig. 1), is plotted in Fig. 2 as a function of the reciprocal temperature. While lowering temperature the precursor amplitude increases, reaching a maximum at about $140 \mathrm{~K}$, then decreases, showing an apparent activation energy of about $7 \mathrm{meV}$, and disappears almost completely at liquid helium temperature. The latter behaviour distinguishes the present precursor from similar features observed at low temperature that were due to the tunnelling via donor states in the well [6].

In DBHs with undoped spacer layer adjacent to the emitter barrier, the electrons accumulated near the barrier under bias conditions cause a band bending that gives rise to the creation of a quasi-triangular quantum well (inset in Fig. 2). 


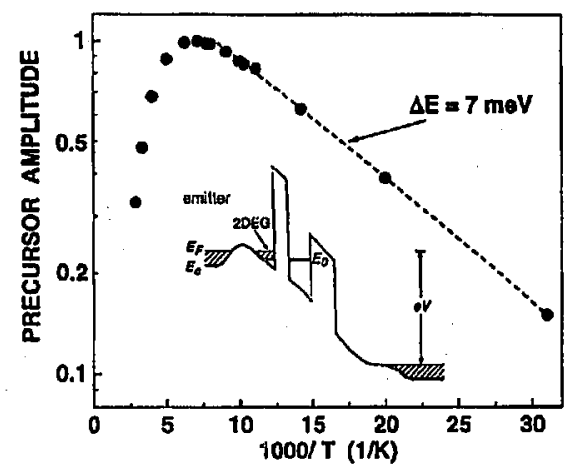

Fig. 2. Logarithm of the precursor amplitude, defined in text, normalized to its maximum value at $140 \mathrm{~K}$ against reciprocal temperature. Least squares fit to the experimental data below $140 \mathrm{~K}$ results in activation energy of $7 \mathrm{meV}$. Inset: schematic diagram of conduction-band edge profile in double-barrier heterostructure having thick spacer layer in emitter region, under bias voltage $V . E_{c}$ and $E_{F}$ are the conduction-band edge and the Fermi level in the emitter, respectively, $E_{0}$ is the subband bottom in the well.

Electron motion perpendicular to the barrier-layer face is then quantized which results in a two-dimensional electron gas (2DEG) occupying subbands within the triangular well. Since $n^{+}$-doped emitter region is separated from the barrier by undoped and lightly-doped layers, an electrostatic bump is formed under bias between this region and the triangular well, as shown schematically in the inset in Fig. 2. In our previous paper [3] we proposed, in line with the above picture and with conclusions of Ref. [2], the following explanation for the resonant part of the $I(V)$ characteristic. The small precursor in front of the main resonance peak is due to the contribution of ballistic electrons that traverse over the bump; this is the 3DEG part of the resonant current. The physical meaning of the observed activation energy is then the difference between the Fermi energy in the emitter region and the energy of the bump top. The electrons surmounting the bump are mainly thermalized in the triangular well but some of them can reach ballistically the barrier, in a part evidenced by the proportion between the precursor and main peak amplitude. Thus, the source of electrons responsible for the main resonance peak is 2DEG in the triangular well.

To verify this model, we performed measurements of the tunnel current and differential conductance, $\mathrm{d} I / \mathrm{d} V$, versus magnetic field, $B$, at fixed bias voltages of the devices. At temperatures below $10 \mathrm{~K}$ both the tunnel current and differential conductance exhibit magneto-tunnelling oscillations which are more pronounced under magnetic field applied parallel to the current. Under bias near that of the onset of main resonance peak the oscillations are periodic when displayed as a function of $1 / B$ (Fig. 3). They can be characterized by a fundamental field, $B_{\mathrm{f}}=$ $[\Delta(1 / B)]^{-1}$, which increases slightly with increasing bias voltage. By tilting the magnetic field at an angle $\theta$ with respect to the normal to the quantum-well plane the fundamental field changes as: $B_{\mathrm{f}}(\theta)=B_{\mathrm{f}}(0) / \cos \theta$ (an example for $\theta=35^{\circ}$ is shown in Fig. 3). This confirms the two-dimensionality of electron 


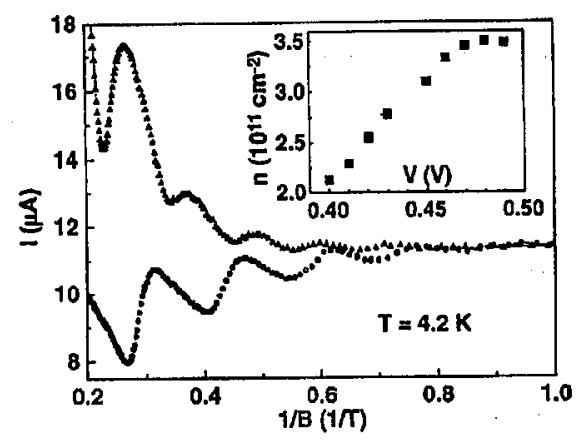

Fig. 3. Magneto-oscillations of the tunnel current against reciprocal magnetic field measured under bias voltage $0.48 \mathrm{~V}$ at a temperature $4.2 \mathrm{~K}$. Circles: magnetic field parallel to the current, triangles: magnetic field tilted at an angle $35^{\circ}$ to the current. Inset shows sheet electron density in the emitter accumulation layer against bias voltage calculated from the fundamental fields of the magneto-oscillations.

gas in the emitter accumulation layer. Note that at low temperatures, at which the magneto-tunnelling oscillations could be measured, the contribution of the precursor to resonant tunnelling was very small.

Nevertheless, in the Fourier transform of the oscillations of differential conductance against $1 / B$, measured at bias voltages below $0.42 \mathrm{~V}$, a weak subsidiary component was revealed which could be related to the 3DEG mode of the resonant tunnelling. Its frequency of about $11 \mathrm{~T}$ was independent of the angle $\theta$ and would correspond to the electron concentration of about $10^{17} \mathrm{~cm}^{-3}$ in the emitter region just before the electrostatic bump (inset in Fig. 2). Sheet electron density in the emitter accumulation layer (of about $3 \times 10^{11} \mathrm{~cm}^{-2}$ ), calculated from the fundamental fields of the main component of magneto-oscillations, against bias voltage is presented in the inset in Fig. 3. Saturation of its values at bias voltages above $0.47 \mathrm{~V}$ clearly indicates the space charge build-up in the quantum well which affects the potential distribution across the structure, as shown schematically in the inset in Fig. 2.

In summary, we have demonstrated the coexistence of two modes of electron gas injection, from 2D and 3D electron gas, in resonant tunnelling through double-barrier heterostructure with an accumulation layer.

\section{Acknowledgments}

The authors are grateful to Drs. M. Kaniewska and K. Regiński (Institute of Electron Technology, Warsaw) for growing the investigated structures. This work was partly supported by the Committee for Scientific Research under grant No. PBZ 28.11/P9.

\section{References}

[1] L. Eaves, G.A. Toombs, F.W. Sheard, C.A. Payling, M.L. Leadbeater, A.S. Alves, T.J. Foster, P.E. Simmonds, M. Henini, O.H. Hughes, J.C. Portal, G. Hill, M.A. Pate, Appl. Phys. Lett. 52, 212 (1988). 
[2] M. Buchanan, H.C. Liu, T.G. Powell, Z.R. Wasilewski, J. Appl. Phys. 68, 4313 (1990).

[3] T. Figielski, T. Wosiński, A. Mąkosa, M. Kaniewska, K. Regiński, Acta Phys. Pol. A 88, 707 (1995).

[4] B.R.A. Neves, J.F. Sampaio, A.S. Alves, M.V.B. Moreira, A.G. de Oliveira, Superlattices Microstr. 20, 181 (1996).

[5] T. Figielski, T. Wosiński, A. Mąkosa, Superlattices Microstr. 24, 69 (1998).

[6] J.G.S. Lok, A.K. Geim, J.C. Maan, I. Marmorkos, F.M. Peeters, N. Mori, L. Eaves, T.J. Foster, P.C. Main, J.W. Sakai, M. Henini, Phys. Rev. B 53, 9554 (1996) and references therein. 